Preprint Version (Green Open Access) of Schüring et al. Reprod Biomed Online 2018; 36(3):294-301.

Doi:10.1016/j.rbmo.2017.11.010

\title{
The endometrial stem cell markers notch-1 and numb are associated with endometriosis
}

Andreas N Schüring ${ }^{a, 1, *}$, Beatrice Dahlhues ${ }^{a, 1}$, Anna Korte ${ }^{a}$, Ludwig Kiesel ${ }^{a}$, Ulf Titze ${ }^{b}$, Birthe Heitkötter ${ }^{\mathrm{b}}$, Christian Ruckert ${ }^{\mathrm{c}}$, Martin Götte ${ }^{\mathrm{a}}$

a Department of Gynaecology and Obstetrics, University Hospital of Münster, 48149 Münster, Germany

${ }^{b}$ Gerhard-Domagk-Institute of Pathology, University Hospital of Münster, 48149 Münster, Germany

${ }^{c}$ Institute of Biometry and Statistics, University Hospital of Münster, 48149 Münster, Germany

* Corresponding author.

E-mail address: Andreas.Schuering@ukmuenster.de (A N Schüring).

${ }^{1}$ These two authors contributed equally to this work.

\section{KEY MESSAGE}

Compared to controls, notch-1 expression in endometrial glands is significantly higher in patients with deep infiltrating endometriosis, whereas numb expression in luminal epithelium is significantly higher in eutopic endometrium of endometriosis patients. Further evaluation of stem cellmarkers notch and numb as biomarkers and therapeutic targets for endometriosis may be worthwhile.

\section{ABSTRACT}

Previous studies reported increased expression of the notch pathway-associated protein Musashi-1 in endometriosis. This case-control study investigates an association of the endometrial stem cell markers notch-1 and numb with endometriosis. Fifty-one endometriosis patients and 76 controls were recruited in the IVF unit and tertiary endometriosis referral centre of a university hospital. All subjects underwent transcervical endometrial biopsy and diagnostic laparoscopy. Expression of endometrial notch-1 and numb was assessed by immunostaining and correlated with clinical data. Association of stem-cell-marker expression with the presence of endometriosis was evaluated. Numb expression in the luminal epithelium was significantly higher in eutopic endometrium of endometriosis patients compared with controls $(20.5 \%$ versus $16.5 \%, \mathrm{P}=0.033)$. Numb-positive single stromal cells were less frequent in endometrioma patients compared with other forms of endometriosis ( 0.3 versus 0.5 cells/visual field; $P=0.028$ ). Notch-1 expression in endometrial glands was significantly higher in patients with deep infiltrating endometriosis compared with controls 
(39.1\% versus $21.8 \% ; \mathrm{P}=0.045)$. We conclude that stem cell markers notch-1 and numb of eutopic endometrium are associated with endometriosis and its clinical presentations, supporting the stem cell hypothesis of endometriosis. These findings could help develop promising research strategies applying endometrial stem cells as novel tools.

\section{Introduction}

Endometriosis, the presence of endometrium outside the uterus, is a disorder with symptoms ranging from infertility and pain to a tumourlike proliferation affecting the integrity of pelvic organs (Giudice and Kao, 2004). Although the pathological mechanisms of endometriosis are still poorly understood, an altered eutopic endometrium has been postulated to constitute the source of the disorder (Sharpe-Timms, 2005). Stem cells have been candidates of interest because of their high proliferation potential and their ability to differentiate into multiple lineages, contributing to characteristic traits of endometriosis (Cervello et al., 2013; Sasson and Taylor, 2008). Adult stem cells have been functionally described in the endometrium obtained by routine transcervical biopsy, providing an easily accessible tool to study a possible contribution of endometrial stem cells to the pathogenesis of endometriosis (Schüring et al., 2011). We could demonstrate that expression of the stem cell marker Musashi-1, a modulator of the notch signalling pathway, is up-regulated in ectopic endometriotic lesions compared with healthy endometrium (Götte et al.,2008). In endometrial carcinoma cells, siRNA-depletion of Musashi-1 resulted in down-regulation of the stem cell marker notch-1 and increased apoptosis (Götte et al., 2011).

The adult stem cell markers notch and numb are evolutionary highly conserved proteins involved in the determination of cell fate. Proteins of the notch family are transmembrane receptors capable of interacting with Delta-like and Jagged ligands, resulting in the formation of an intracellular domain that regulates nuclear gene transcription (Talora et al., 2008). Notch signalling constitutes a central mechanism of multicellular development by controlling maintenance and fate of stem cells. The signal conveyed by the notch receptor is pleiotropic and context-specific, affecting numerous physiological processes of proliferation, differentiation and apoptosis (Weijzen et al., 2002). Notch malfunction has been associated with human disease and is evaluated as a potential target for cancer therapy (Ibrahim et al., 2017; Pannuti et al., 2010; Wu et al., 2010). Similar to notch, numb plays a critical role in the promotion of cell fate, contributing both to physiological cell development and proliferating diseases, e.g. neoplasms (Pece et al., 2004). Exerting a complex pattern of functions, numb can context-specifically suppress notch action and act as its functional antagonist. Numb is involved in the proteolytic degradation of notch via modulation of endocytosis, thus regulating notch-mediated signalling (McGill and McGlade, 2003; McGill et al., 2009). Given the emerging role of endometrial stem cells in the pathogenesis of endometriosis and their potential relevance as a future therapeutic target, we evaluated the possible role of notch-1 and numb in eutopic endometrium of patients with endometriosis compared with healthy controls.

\section{Materials and methods}

\section{Patients}


Written informed consent was obtained from all patients included in the study, which was carried out according to the principles of the Helsinki Convention and approved by the local ethics committee (1 IX Greb, from 2001-09-19). In a university-based IVF unit, female patients undergoing standardized diagnostics prior to assisted reproductive techniques were subject to transcervical endometrial biopsy as published previously (Schüring et al., 2011). Briefly, endometrial biopsies were obtained between cycle days 22 and 24 with a Probet catheter (Gynemed, Lensahn, Germany). Exclusively patients undergoing diagnostic laparoscopy in the tertiary endometriosis referral centre of the university were included in the study. Operative procedures were performed by specialized fertility surgeons, who classified endometriosis according to the ASRM criteria (American Society for Reproductive Medicine, 1997). Clinical diagnosis was histopathologically confirmed by an experienced pathologist. To exclude a confounding influence by different cycle phases in patients with ovulatory disorders, endometrium was staged according to established criteria (Noyes et al., 1950). Anthropometric and clinical data were obtained from the patient records.

\section{Immunohistochemistry}

Sample collection followed the BRISQ Tier 1 requirements (Simeon-Dubach et al., 2012). Endometrial tissue was fixed in $10 \%$ formalin and embedded in paraffin using standard procedures, and documented at the Department of Pathology of Münster University Hospital. Paraffin blocks were stored at ambient temperature for less than 7 years. Consecutive $3 \mathrm{~m}$ sections were cut from paraffin blocks and placed on poly-L-lysine-coated coverslips. Dried coverslips were deparaffinized, rehydrated and treated with target retrieval solution (pH 6.1, Dako, Glostrup, Denmark) for 35 min in a steamer, followed by three washes in phosphate-buffered saline (PBS). Sections were blocked with peroxidase (Dako), followed by a second block with 10\% Aurion BSA (Dako) for $30 \mathrm{~min}$. Sections were then washed in PBS and incubated with numb antibodies (1:70, rabbit polyclonal IgG, Santa Cruz Biotechnology) or with notch-1 antibodies (1:50, rabbit polyclonal IgG, Santa Cruz Biotechnology) diluted with Dako Real Antibody diluent, for $16 \mathrm{~h}$ at $4^{\circ} \mathrm{C}$. Normal rabbit immunoglobulin fraction (\# X0903, Dako) was diluted to the same protein concentration as the respective notch/numb rabbit polyclonal IgGs and served as a negative control. After three washes, primary antibodies were detected using anti-rabbit EnVision systems and AEC-substrate chromogen (7 min) according to the manufacturer (Dako). Sections were counterstained with Mayers haemalum (Merck, Darmstadt, Germany) and embedded in Kaiser's glycerol gelatin (Merck).

\section{Microscopic evaluation}

Microscopic evaluation was performed by three independent investigators (BD, MG, AK) blinded for the patient's diagnosis using an Axiophot 100 microscope, equipped with a CCD camera and Axiovision Software (Zeiss, Göttingen, Germany). Staining intensity was evaluated at $20 \times$ magnification for five visual fields $(700 \mu \mathrm{m} \times 525 \mu \mathrm{m})$ per stained section. The stromal staining, a 
diffuse cytoplasmic staining of the glands and a diffuse cytoplasmic staining of the luminal epithelium were assessed according to the following score: 0: weak staining, 1: medium staining, 2: intense staining. Distinct membranous staining of the glands and the luminal epithelium were assessed as a percentage, ranging from $0 \%$ (lowest intensity) to $100 \%$ (highest intensity). In addition, markerpositive stromal cell nests and single stromal cells were counted and expressed as mean cell number per visual field. Only samples with unambiguous staining results were included in the final analysis. Thirteen samples were excluded due to the presence of an ambiguous gradient-like staining pattern.

\section{Statistical analysis}

Statistical analysis was performed using SPSS 18 software (SPSS, Chicago, USA). Comparing stem cell marker expression between groups, the Mann-Whitney U-test and Fisher's exact test were applied. Spearman's correlation was used to analyse correlations between notch-1 and numb in the different groups. A P-value $<0.05$ was considered as statistically significant.

\section{Results}

\section{Patient characteristics}

One hundred and twenty-seven patients of the infertility unit who underwent endometrial biopsy and diagnostic laparoscopy were finally included in this study after selection of samples with unambiguous results. Patient characteristics are given in Table 1. Fiftyone patients were diagnosed with endometriosis, while 76 individuals served as controls after endometriosis had been excluded during laparoscopy. No significant differences were observed in patients with endometriosis when compared with controls in respect to age, menstrual history and cycle day (Table 1). Patients in the control group displayed a higher BMI (24.8 versus $23.3 ; \mathrm{P}=0.019$ ) and a higher incidence of preceding pregnancies $(0.6$ versus $0.2 ; \mathrm{P}=0.017$ ). The ASRM stages were distributed as follows: ASRM I, 25 patients (49\%), ASRM II, 11 patients (22\%), ASRM III, 10 patients (20\%) and ASRM IV, 5 patients (10\%). The phenotypic presentation of the disorder was distributed as follows: forty-eight cases of peritoneal endometriosis represented the most frequent phenotype of the disease. An endometrioma was present in 15 patients and a deep infiltrating endometriosis in 15 cases. In 22 patients, the entities of the disease occurred in combinations. While peritoneal endometriosis was found as the sole presentation in 28 patients, it was also observed in combination with deep infiltrating endometriosis $(n=8)$ or with an endometrioma $(n=8)$. In four patients with severe endometriosis, a combination of all three entities of the disease was observed. The combination of an endometrioma with deep infiltrating endometriosis occurred in two cases and the sole presentation of an endometrioma was found in one patient. Deep infiltrating endometriosis as a sole manifestation of the disease was not observed in our study population (Table 2).

\section{Staining pattern of notch-1 and numb}


A similar staining pattern was observed for notch-1 (Figure 1) and numb (Figure 2) in the assessed endometrial samples. Compared with the negative control (Figures $1 \mathrm{E}$ and $2 \mathrm{E}$ ), both stem cell markers displayed a stromal staining (Figures $1 \mathrm{~A}$ and $2 \mathrm{~A}$ ), a diffuse cytoplasmic staining of the glands (Figures $1 \mathrm{~B}$ and $2 \mathrm{~B}$ ) and a diffuse cytoplasmic staining of the luminal epithelium (Figures $1 \mathrm{C}$ and $2 \mathrm{C}$ ). Furthermore, we observed a staining of stromal cell nests (Figures 1D and 2D) and of stromal single cells (Figures $1 \mathrm{H}, 1 \mathrm{~A}$ and $2 \mathrm{H}$, arrowheads). Besides, both markers displayed a distinct membranous staining of the glands (Figures $1 \mathrm{~F}$ and $2 \mathrm{~F}$ ) and a distinct membranous staining of the luminal epithelium (Figures 1G and 2G).

\section{Numb and notch-1 expression compared between groups}

Endometrial numb expression in the distinct membranous staining of luminal epithelium was significantly higher in patients with endometriosis compared with controls $(20.5 \%$ versus $16.5 \%$; $P=$ 0.033 , Figure $3 \mathrm{~A}$ ) and a corresponding difference was observed for subjects with peritoneal endometriosis compared with controls (19.9\% versus $16.5 \%$; $\mathrm{P}=0.046$, Figure $3 \mathrm{~B})$. When comparing notch-1 expression, membranous staining of endometrial glands was significantly higher in patients with deep infiltrating endometriosis compared with controls (39.1\% versus $21.8 \% ; \mathrm{P}=0.045$ ) (Figure 3C). Additionally, numb staining of single stromal cells was less pronounced in patients with an endometrioma compared with endometriosis patients with peritoneal or deep infiltrating endometriosis $(0.3 \pm 0.1$ cells/visual field versus $0.5 \pm 0.1$ cells/visual field; mean $\pm S E M ; P=0.028)$.

\section{Mutual correlations between notch-1 and numb}

Because numb can antagonize the notch pathway (McGill et al., 2009), we compared the mutual correlation of stem cell markers in different groups. In the control group, significant mutual correlations of notch-1 with numb were found for the stromal staining (correlation coefficient $\rho=$ $0.551, P<0.001)$, the distinct membranous glandular staining $(\rho=0.660, P<0.001)$, the diffuse cytoplasmic glandular staining $(\rho=0.593, P<0.001)$, the diffuse cytoplasmic staining of luminal epithelium ( $\rho=0.491, P<0.033)$ and for the stromal cell nests $(\rho=0.205, P=0.034)$. The staining of stromal single cells and the distinct membranous staining of the luminal epithelium did not display correlations between notch-1 and numb expression. In contrast to healthy controls, patients with endometriosis displayed fewer mutual correlations between notch-1 and numb. While correlations between these markers were similar to controls with respect to stromal staining $\rho=0.683, P<$ $0.001)$, distinct membranous glandular $(\rho=0.547, P<0.001)$ and cytoplasmic glandular staining $(\rho=$ $0.650, P<0.001$ ), while no correlation for the diffuse cytoplasmic staining of luminal epithelium was observed. This finding was confirmed in subjects with peritoneal endometriosis, endometriotic cysts and deep infiltrating endometriosis. In addition, in subjects with deep infiltrating endometriosis no correlation between notch-1 and numb in the cytoplasmic glandular staining was observed.

\section{Discussion}

This study reports a significant association of endometrial notch-1 and numb with endometriosis, adding novel information on a possible role of stem cells in the aetiology of the disorder. We find 
that endometriosis patients display a different expression of stem cell markers in their eutopic endometrium when compared with healthy controls. Endometrial expression of notch-1 and numb also discriminated between different phenotypic manifestations of endometriosis. In addition, mutual correlations of notch-1 with numb were decreased in endometriosis patients compared with controls.

A strength of the study is that it analyses a population of adequate sample size that has been thoroughly characterized under standardized conditions. Subjects with proven endometriosis were compared with an identically assessed control group, in which absence of disease was confirmed by laparoscopy. A potential drawback is that it cannot be completely excluded that the phenotypic heterogeneity of endometriosis with a partial overlap in the study individuals has increased the risk of a confounding influence. In future, evaluation of large, well-characterized populations assessed by minimally invasive endometrial biopsy appears to be a promising tool to confirm and extend the present findings in high throughput collectives.

While adult stem cells are postulated to be involved in the pathogenesis of endometriosis, little information on stem cell markers in eutopic endometrium, the potential source of dislocated endometrial implants, is available to date. Initial data were reported on notch-1 (Afshar et al., 2012; Cobellis et al., 2008; Mitsuhashi et al., 2012; Su et al., 2015) and numb (Afshar et al., 2012) in physiological and pathological conditions of the endometrium.

In normal endometrium of mice and primates notch appears to be associated with the establishment of a functioning luteal phase. This is supported by some studies in which notch was variably regulated throughout the menstrual cycle (Afshar et al., 2012; Cobellis et al., 2008). However, in another study, no change in endometrial notch receptor expression during the menstrual cycle was observed (Mitsuhashi et al., 2012). In accordance with the latter findings, the present study did not detect a cycle-dependent expression of notch-1 and numb when comparing proliferative with secretory endometrium or when evaluating endometrial dating and serum progesterone as continuous markers of luteal transition (data not shown).

Particularly striking is the observation by some authors that notch-1 in primate endometrium was increased during the luteal window of uterine receptivity (Afshar et al., 2012). Additionally, secretory phase notch-1 was up-regulated by chorionic gonadotrophin in combination with oestrogen and progesterone, whereas progesterone increases intracellular notch-1, which can modulate transcription in a complex with progesterone receptor. Notch inhibition prevented decidualization. While these data provide a functional link between notch-1 and fertility in primates, we can only speculate whether the altered expression pattern of endometrial notch-1 observed in individuals of this study presenting with deep infiltrating endometriosis is associated with altered fertility or progesterone resistance, postulated to play a role in the aetiology of the disorder (Kim et al., 2013). An inhibitory effect of the notch pathways on decidualization was recently supported by functional data on endometrial stroma cells derived from endometriosis patients and controls (Su et al., 2015). Surprisingly, in contrast to our study, Su et al. (2015) reported that several components of the notch pathway, including notch-1, were down-regulated in the eutopic endometrium of endometriosis patients. While differences in patient collectives may be one underlying reason for this discrepancy, we want to stress that the group size in our immunohistochemical study comprised over 50 patients, whereas Su et al. (2015) only included six patients/experimental group. 
genesis in various human neoplasms (Cuevas et al., 2005; Miyamoto et al., 2003; Steg et al., 2011). In endometrial cancer, notch-1 expression is associated with particularly invasive properties, such as vessel or lymph node involvement and myometrial invasion (Mitsuhashi et al., 2012). These data are in accordance with our previous finding that down-regulation of Musashi-1 in endometrial carcinoma cells leads to reduced notch-1 expression and induction of apoptosis in malignant cells (Götte et al., 2011). Overall these associations are in accordance with the correlation of an increased membranous notch-1 staining of endometrial glands in patients with deep infiltrating endometriosis in the present study. Up-regulation of notch-1 in the eutopic endometrium may facilitate the establishment of particularly invasive ectopic lesions in these individuals.

Recent data on endometrial numb are suggestive of a physiological antagonism to notch-1 in the luteal phase (Afshar et al., 2012). In the baboon model, numb expression increased when notch-1 decreased during decidualization. When relating endometrial notch-1 and numb in our study, we did not observe a complementary antagonism as has been described before, but rather a parallel expression pattern of the two markers: when notch-1 was elevated numb was also increased. We postulate that numb up-regulation might reflect a context-specific compensatory mechanism to counteract increased notch-1 activation. Moreover, notch-1 and numb expression are modulated by additional factors, including the RNA binding protein Musashi-1, which was previously shown to be dysregulated in endometriosis (Götte et al., 2008), and by the co-expression pattern of notch ligands. Notably, in the present study, mutual correlations between notch-1 and numb staining patterns were decreased in endometriosis patients, especially in subjects with deep infiltrating endometriosis. It could be speculated that the observed disbalance of the two antagonistic stem cell markers contributed to the pathogenesis of endometriosis. While a high expression of notch-1 would be balanced by a high expression of numb in the healthy individual, in endometriosis this balance is lost.

We postulate that the observed differential expression of endometrial notch-1 and numb in endometriosis and its subgroups could indicate a varying pathological mechanism underlying distinct phenotypes of the disorder and involving stem cell function (Nisolle and Donnez, 1997). Furthermore, studied stem cell markers might offer future diagnostic potential to be used in a test for endometriosis applying minimally invasive endometrial biopsy as proposed before (May et al., 2011; Schüring et al., 2011). In the context of this pilot study it could be speculated that while luminal numb expression may rather be associated with the presence of endometriosis in general, increased glandular notch-1 expression could more specifically help to indicate the manifestation of deep infiltrating endometriosis in affected subjects. In addition, a reduced numb expression in single stromal cells could point to an ovarian manifestation of endometriosis. However, although our results reveal promising associations, because of a partial phenotypic overlap in endometriosis subgroups it is too early to conclude a diagnostic eligibility of endometrial stem cell markers and further studies are warranted to confirm these findings. Finally, results of this study are supportive of the hypothesis that stem cells and their properties could become promising future therapeutic targets broadening treatment strategies of endometriosis.

\section{Acknowledgements}

This study was supported by EU-H2020-RISE [grant no. 691058 MOMENDO] (to M.G.) and a Bayer HealthCare Focus grant (to M.G). We thank Mrs Silke Brockhoff for organization of the sample database and Peter Barth for discussions. 
Declaration: The authors report no financial or commercial conflicts of interest.

\section{Keywords:}

Adult stem cells

Endometriosis

Endometrium

Notch

Numb

\section{REFERENCES}

Afshar, Y., Miele, L., Fazleabas, A.T., 2012. Notch1 is regulated by chorionic gonadotrophin and progesterone in endometrial stromal cells and modulates decidualization in primates. Endocrinology $153,2884-2896$.

American Society for Reproductive Medicine, 1997. Revised American Society for Reproductive Medicine classification of endometriosis. Fertil. Steril. 67, 817-821.

Cervello, I., Mas, A., Gil-Sanchis, C., Simón, C., 2013. Somatic stem cells in the human endometrium. Semin. Reprod. Med. 31, 69-76.

Cobellis, L., Caprio, F., Trabucco, E., Mastrogiacomo, A., Coppola, G., Manente, L., Colacurci, N., De Falco, M., De Luca, A., 2008. The pattern of expression of Notch protein members in normal and pathological endometrium. J. Anat. 213, 464-472.

Cuevas, I.C., Slocum, A.L., Jun, P., Costello, J.F., Bollen, A.W., Riggins, G.J., McDermott, M.W., Lal, A., 2005. Meningioma transcript profiles reveal deregulated Notch signaling pathway. Cancer Res. 65, 5070-5075.

Giudice, L.C., Kao, L.C., 2004. Endometriosis. Lancet 364, 1789-1799.

Götte, M., Wolf, M., Staebler, A., Buchweitz, O., Kelsch, R., Schüring, A.N., Kiesel, L., 2008. Increased expression of the adult stem cell marker Musashi-1 in endometriosis and endometrial carcinoma. J. Pathol. 215, 317-329.

Götte, M., Greve, B., Kelsch, R., Müller-Uthoff, H., Weiss, K., Kharabi Masouleh, B., Sibrowski, W., Kiesel, L., Buchweitz, O., 2011. The adult stem cell marker musashi-1 modulates endometrial carcinoma cell cycle progression and apoptosis via notch-1 and p21(WAF1/CIP1). Int. J. Cancer 129, 2042-2049. 
Ibrahim, S.A., Gadalla, R., El-Ghonaimy, E.A., Samir, O., Mohamed, H.T., Hassan, H., Greve, B., ElShinawi, M., Mohamed, M.M., Götte, M., 2017. Syndecan-1 is a novel molecular marker for triple negative inflammatory breast cancer and modulates the cancer stem cell phenotype via the IL6/STAT3, Notch and EGFR signaling pathways. Mol. Cancer 16, 57.

Kim, J.J., Kurita, T., Bulun, S.E., 2013. Progesterone action in endometrial cancer, endometriosis, uterine fibroids, and breast cancer. Endocr. Rev. 34, 130-162.

May, K.E., Villar, J., Kirtley, S., Kennedy, S.H., Becker, C.M., 2011. Endometrial alterations in endometriosis: a systematic review of putative biomarkers. Hum. Reprod. Update 17, 637-653.

McGill, M.A., McGlade, C.J., 2003. Mammalian numb proteins promote Notch1 receptor ubiquitination and degradation of the Notch1 intracellular domain. J. Biol. Chem. 278, 23196-23203.

McGill, M.A., Dho, S.E., Weinmaster, G., McGlade, C.J., 2009. Numb regulates post-endocytic trafficking and degradation of Notch1. J. Biol. Chem. 284, 26427-26438.

Mitsuhashi, Y., Horiuchi, A., Miyamoto, T., Kashima, H., Suzuki, A., Shiozawa, T., 2012. Prognostic significance of Notch signaling molecules and their involvement in the invasiveness of endometrial carcinoma cells. Histopathology 60, 826-837.

Miyamoto, Y., Maitra, A., Ghosh, B., Zechner, U., Argani, P., lacobuzio-Donahue, C.A., Sriuranpong, V., Iso, T., Meszoely, I.M., Wolfe, M.S.,Hruban, R.H., Ball, D.W., Schmid, R.M., Leach, S.D., 2003.

Notch mediates TGF alpha-induced changes in epithelial differentiation during pancreatic tumourigenesis. Cancer Cell 3, 565-576.

Nisolle, M., Donnez, J., 1997. Peritoneal endometriosis, ovarian endometriosis, and adenomyotic nodules of the rectovaginal septum are three different entities. Fertil. Steril. 68, 585-596.

Noyes, R.W., Hertig, A.T., Rock, J., 1950. Dating the endometrial biopsy. Fertil. Steril. 1, 3-25.

Pannuti, A., Foreman, K., Rizzo, P., Osipo, C., Golde, T., Osborne, B., Miele, L., 2010. Targeting Notch to target cancer stem cells. Clin. Cancer Res. 16, 3141-3152.

Pece, S., Serresi, M., Santolini, E., Capra, M., Hulleman, E., Galimberti, V., Zurrida, S., Maisonneuve, P., Viale, G., Di Fiore, P.P., 2004. Loss of negative regulation by Numb over Notch is relevant to human breast carcinogenesis. J. Cell Biol. 167, 215-221.

Sasson, I.E., Taylor, H.S., 2008. Stem cells and the pathogenesis of endometriosis. Ann. N. Y. Acad. Sci. $1127,106-115$.

Schüring, A.N., Schulte, N., Kelsch, R., Röpke, A., Kiesel, L., Götte, M., 2011. Characterization of endometrial mesenchymal stem-like cells obtained by endometrial biopsy during routine diagnostics. Fertil. Steril. 95, 423-426.

Sharpe-Timms, K.L., 2005. Defining endometrial cells: the need for improved identification at ectopic sites and characterization in eutopic sites for developing novel methods of management for endometriosis. Fertil. Steril. 84, 35-37. 
Simeon-Dubach, D., Burt, A.D., Hall, P.A., 2012. Quality really matters: the need to improve specimen quality in biomedical research. J. Pathol. 228, 431-433.

Steg, A.D., Katre, A.A., Goodman, B., Han, H.D., Nick, A.M., Stone, R.L., Coleman, R.L., Alvarez, R.D., Lopez-Berestein, G., Sood, A.K., Landen, C.N., 2011. Targeting the notch ligand JAGGED1 in both tumour cells and stroma in ovarian cancer. Clin. Cancer Res. 17, 5674-5685.

Su, R.W., Strug, M.R., Joshi, N.R., Jeong, J.W., Miele, L., Lessey, B.A., Young, S.L., Fazleabas, A.T., 2015. Decreased Notch pathway signaling in the endometrium of women with endometriosis impairs decidualization. J. Clin. Endocrinol. Metab. 100, E433-E442.

Talora, C., Campese, A.F., Bellavia, D., Felli, M.P., Vacca, A., Gulino, A., Screpanti, I., 2008. Notch signaling and diseases: an evolutionary journey from a simple beginning to complex outcomes. Biochim. Biophys. Acta 1782, 489-497.

Weijzen, S., Rizzo, P., Braid, M., Vaishnav, R., Jonkheer, S.M., Zlobin, A., Osborne, B.A., Gottipati, S., Aster, J.C., Hahn, W.C., Rudolf, M., Siziopikou, K., Kast, W.M., Miele, L., 2002. Activation of Notch-1 signaling maintains the neoplastic phenotype in human Ras transformed cells. Nat. Med. 8, 979-986.

Wu, Y., Cain-Hom, C., Choy, L., Hagenbeek, T.J., de Leon, G.P., Chen, Y., Finkle, D., Venook, R., Wu, X., Ridgway, J., Schahin-Reed, D., Dow, G.J., Shelton, A., Stawicki, S., Watts, R.J., Zhang, J., Choy, R., Howard, P., Kadyk, L., Yan, M., Zha, J., Callahan, C.A., Hymowitz, S.G., Siebel, C.W., 2010. Therapeutic antibody targeting of individual Notch receptors. Nature 464, 1052-1057.

Figures and tables:

Table 1 - Clinical data of patients and controls.

\begin{tabular}{|c|c|c|c|c|}
\hline & $\begin{array}{l}\text { All patients } \\
\mathrm{n}=127\end{array}$ & $\begin{array}{l}\text { Endometriosis } \\
n=51\end{array}$ & $\begin{array}{l}\text { Controls } \\
n=76\end{array}$ & $P$-value \\
\hline Age (years) & $\begin{array}{l}33.4 \pm 4.3 \\
(21-44)\end{array}$ & $\begin{array}{l}33.0 \pm 4.1 \\
(22-39)\end{array}$ & $\begin{array}{l}33.4 \pm 4.4 \\
(21-44)\end{array}$ & NS \\
\hline $\mathrm{BMI}(\mathrm{kg} / \mathrm{m} 2)$ & $\begin{array}{l}24.3 \pm 4.3 \\
(17.6-39.0)\end{array}$ & $\begin{array}{l}23.3 \pm 3.3 \\
(17.9-29.6)\end{array}$ & $\begin{array}{l}24.8 \pm 4.6 \\
(17.6-39.0)\end{array}$ & 0.019 \\
\hline Gravida & $\begin{array}{l}0.5 \pm 1.0 \\
(0-5)\end{array}$ & $\begin{array}{l}0.2 \pm 0.7 \\
(0-4)\end{array}$ & $\begin{array}{l}0.6 \pm 1.1 \\
(0-5)\end{array}$ & 0.017 \\
\hline Para & $\begin{array}{l}0.1 \pm 0.4 \\
(0-2)\end{array}$ & $\begin{array}{l}0.1 \pm 0.2 \\
(0-1)\end{array}$ & $\begin{array}{l}0.2 \pm 0.5 \\
(0-2)\end{array}$ & NS \\
\hline $\begin{array}{l}\text { Menstrual cycle } \\
\text { interval (days) }\end{array}$ & $\begin{array}{l}31.7 \pm 15.5 \\
(24-150)\end{array}$ & $\begin{array}{l}28.9 \pm 4.8 \\
(24-59)\end{array}$ & $\begin{array}{l}33.1 \pm 18.3 \\
(24-150)\end{array}$ & NS \\
\hline $\begin{array}{l}\text { Clinical cycle day } \\
\text { (days) }\end{array}$ & $\begin{array}{l}22.3 \pm 2.0 \\
(8-28)\end{array}$ & $\begin{array}{l}21.7 \pm 2.7 \\
(8-28)\end{array}$ & $\begin{array}{l}22.5 \pm 1.5 \\
(19-26)\end{array}$ & NS \\
\hline
\end{tabular}




\begin{tabular}{|c|c|c|c|c|}
\hline $\begin{array}{l}\text { Histological cycle } \\
\text { day (days) }\end{array}$ & $\begin{array}{l}18.3 \pm 4.7 \\
(4.0-25.5)\end{array}$ & $\begin{array}{l}18.6 \pm 4.4 \\
(5.5-24.0)\end{array}$ & $\begin{array}{l}18.2 \pm 4.9 \\
(4.0-25.5)\end{array}$ & NS \\
\hline $\begin{array}{l}\text { Progesterone } \\
(\mathrm{ng} / \mathrm{ml})\end{array}$ & $\begin{array}{l}9.8 \pm 7.4 \\
(0.2-29.3)\end{array}$ & $\begin{array}{l}10.8 \pm 7.5 \\
(0.2-25.3)\end{array}$ & $\begin{array}{l}9.3 \pm 7.4 \\
(0.2-29.3)\end{array}$ & NS \\
\hline \multicolumn{5}{|c|}{$\begin{array}{l}\text { Data are presented as mean } \pm 2 \text { SD (standard deviation) and range. Endometriosis patients were } \\
\text { compared with controls by applying the Mann-Whitney U-test, } \mathrm{P}<0.05 \text { was considered statistically } \\
\text { significant. } \\
\mathrm{BMI}=\text { body mass index; NS = not statistically significant. }\end{array}$} \\
\hline
\end{tabular}

Table 2 - Phenotypic presentations of endometriosis.

\begin{tabular}{|l|l|l|l|l|l|l|}
\hline $\begin{array}{l}\text { Peritoneal } \\
\text { endometriosis }\end{array}$ & $\begin{array}{l}\text { Peritoneal } \\
\text { endometriosis } \\
+ \\
\text { deep } \\
\text { endometriosis }\end{array}$ & $\begin{array}{l}\text { Peritoneal } \\
\text { endometriosis } \\
+\end{array}$ & $\begin{array}{l}\text { Peritoneal } \\
\text { endometriosis } \\
+\end{array}$ & $\begin{array}{l}\text { Deep } \\
\text { endometriosis } \\
+ \\
\text { endometrioma } \\
\text { endoep } \\
\text { endometriosis } \\
+\end{array}$ & $\begin{array}{l}\text { Endometrioma } \\
\text { endometrioma }\end{array}$ & $\begin{array}{l}\text { Deep } \\
\text { endometriosis }\end{array}$ \\
\hline $\mathbf{n = 2 8}$ & $\mathbf{n = 8}$ & $\mathbf{n = 8}$ & $\mathbf{n = 4}$ & $\mathbf{n = 2}$ & $\mathbf{n = 1}$ & $\mathbf{n = 0}$ \\
\hline
\end{tabular}



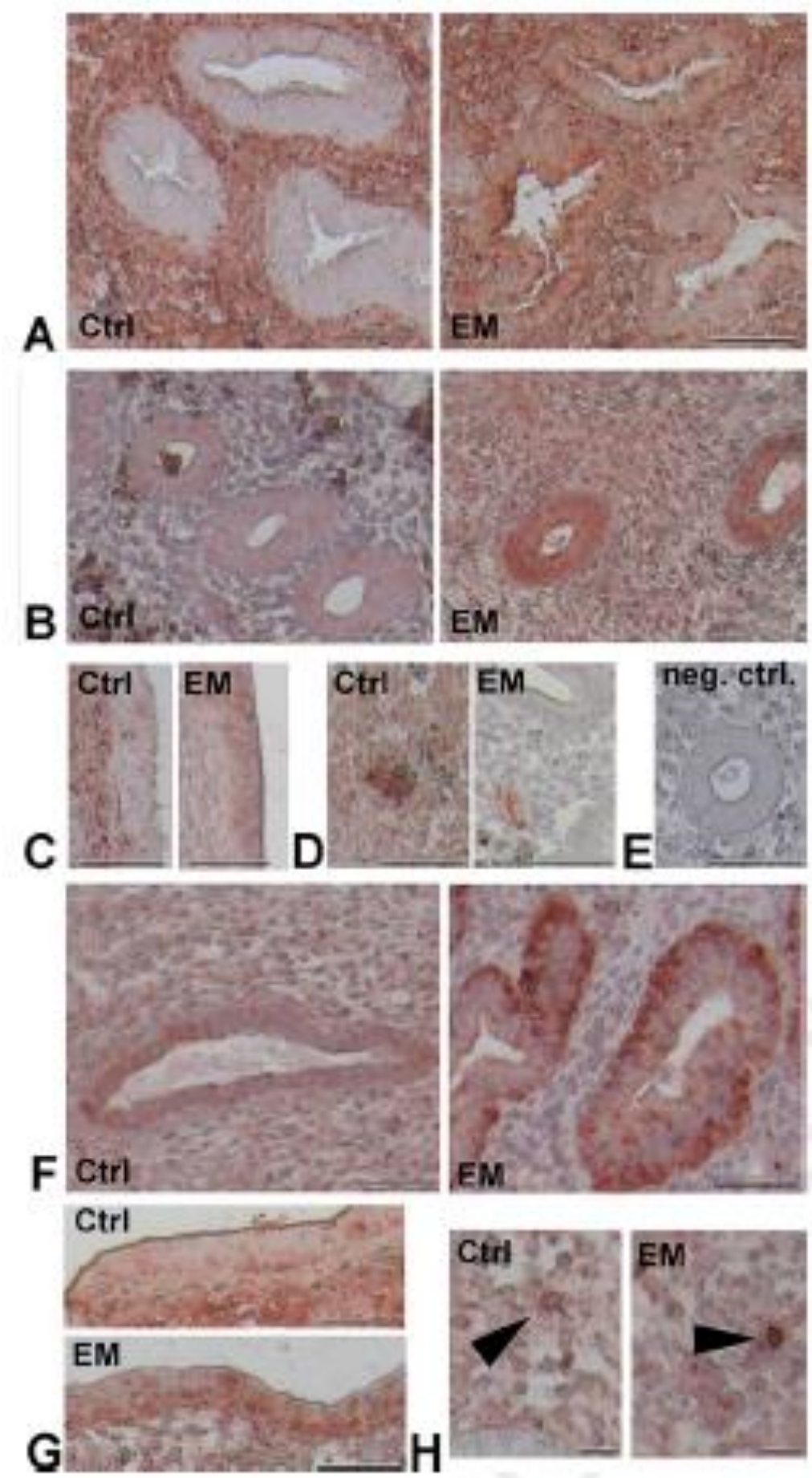

Figure 1 - Immunohistochemical staining pattern of notch-1. Notch-1 expression was evaluated in the eutopic endometrium of endometriosis patients (EM) and endometriosis-free controls (Ctrl). Notch-1 displayed a stromal staining (Figure 1A), a diffuse cytoplasmic staining of the glands (Figure 1B) and a diffuse cytoplasmic staining of the luminal epithelium (Figure 1C). Furthermore, we observed a staining of stromal cell nests (Figure 1D) and of stromal single cells (Figure $1 \mathrm{H}$, arrowheads). Besides, notch-1 displayed a distinct membranous staining of the glands (Figure 1F) and a distinct membranous staining of the luminal epithelium (Figure 1G). Figure 1E shows a technical negative control of a control patient, in which the primary antibody was replaced with pre-immune rabbit IgG, and counterstained. A technical negative control for an EM patient is shown in Figure 2E. Scale bars represent $50 \mu \mathrm{m}$ for Figure $1 \mathrm{~A}-\mathrm{G}$ and $20 \mu \mathrm{m}$ for Figure $1 \mathrm{H}$. 


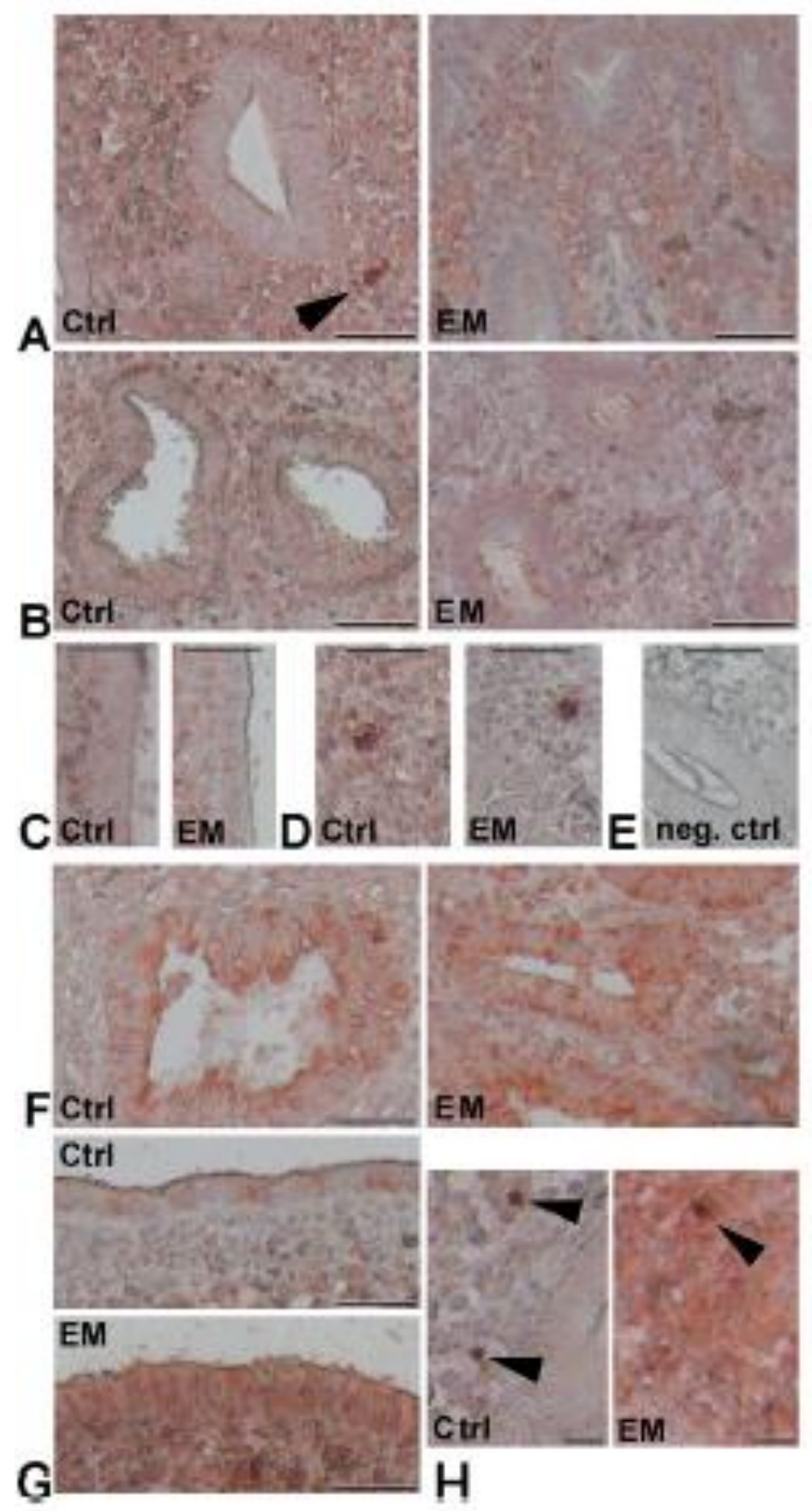

Figure 2 - Immunohistochemical staining pattern of numb. Numb expression was evaluated in the eutopic endometrium of endometriosis patients (EM) and endometriosis-free controls (Ctrl). Numb displayed a stromal staining (Figure 2A), a diffuse cytoplasmic staining of the glands (Figure 2B) and a diffuse cytoplasmic staining of the luminal epithelium (Figure $2 \mathrm{C}$ ). Furthermore, we observed a staining of stromal cell nests (Figure 2D) and of stromal single cells (Figure 2A, H, arrowheads). Besides, numb displayed a distinct membranous staining of the glands (Figure $2 \mathrm{~F}$ ) and a distinct membranous staining of the luminal epithelium (Figure 2G). Figure 2E shows a technical negative control of an EM patient, in which the primary antibody was replaced with pre-immune rabbit IgG, and counterstained. A technical negative control for a control individual is shown in Figure 1E. Scale bars represent $50 \mu \mathrm{m}$ for Figure $2 \mathrm{~A}-\mathrm{G}$ and $20 \mu \mathrm{m}$ for Figure $2 \mathrm{H}$. 

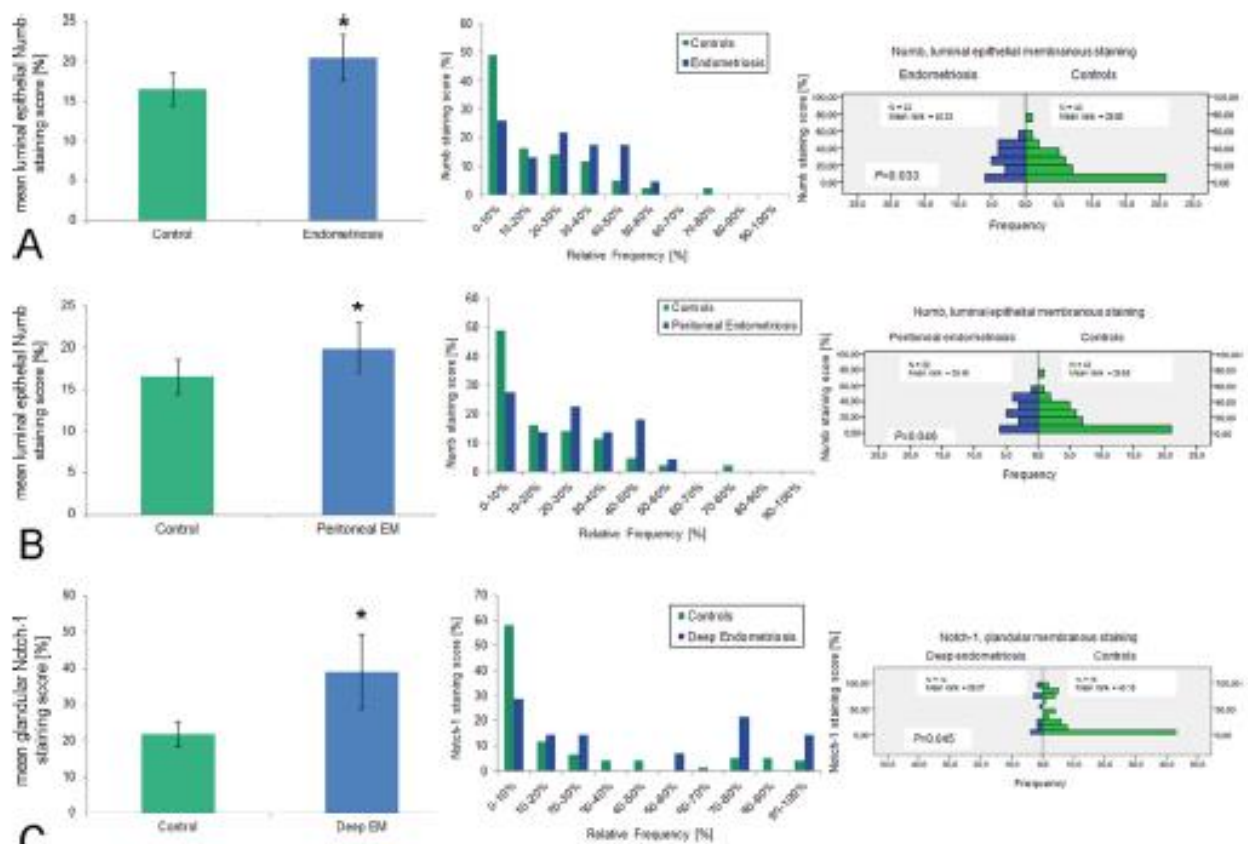

Figure 3 - Endometrial stem cell marker expression compared between groups. Stem cell marker expression in eutopic endometrial samples of patients with endometriosis compared with healthy controls applying the Mann-Whitney U-test (Figure 3A-C). A and B include samples which included luminal epithelium only. $C$ includes all deep endometriosis cases. Numb expression was significantly higher in the luminal epithelium of patients with endometriosis (Figure $3 \mathrm{~A}$ ) and with peritoneal endometriosis (Figure 3B) compared with controls. Notch-1 expression was significantly pronounced in patients with deep infiltrating endometriosis compared with controls (Figure 3C). Left panels: Mean staining score \pm SEM. ${ }^{*} P<0.05$. Central panels: Frequency bar plot. Right panels: Display of statistical analysis. In the samples containing luminal epithelium with positive numb staining, phenotypic manifestations of endometriosis were distributed as follows: Exclusively peritoneal $(n=$ 12), peritoneal + deep infiltrating $(n=6)$, peritoneal + endometrioma $(n=2)$, peritoneal + deep infiltrating + endometrioma $(n=2)$, deep infiltrating + endometrioma $(n=1)$. 